(C) 2015 IEEE. Personal use of this material is permitted. Permission from IEEE must be obtained for all other uses, in any current or future media, including reprinting/republishing this material for advertising or promotional purposes, creating new collective works, for resale or redistribution to servers or lists, or reuse of any copyrighted component of this work in other works. 


\title{
Energy-Efficient Distributed Beamforming in UWB Based Implant Body Area Networks
}

\author{
Jie Ding*, Eryk Dutkiewicz*, Xiaojing Huang ${ }^{\dagger}$, Gengfa Fang* \\ *Department of Engineering, Macquarie University, Australia \\ \{jie.ding1, eryk.dutkiewicz, gengfa.fang\}@mq.edu.au \\ $\dagger$ University of Technology, Sydney \\ Xiaojing.Huang@uts.edu.au
}

\begin{abstract}
In this paper, we investigate a distributed beamforming problem to optimize energy efficiency (EE) in ultra-wideband (UWB) based implant body area networks (IBANs). To evaluate the impact of relay location on the EE, a relay location based cooperative network model is proposed, where multiple on-body relays are employed to assist an implant node to communicate with a BAN coordinator. With the proposed model, the EE optimization problem is mathematically formulated as a nonconvex optimization problem. Sequential quadratic programming (SQP) combined with scatter search are applied to find the corresponding optimal solution. Simulation results illustrate that the proposed beamforming scheme outperforms other transmission schemes. A remarkable improvement can be achieved not only in EE but also in spectral efficiency (SE) compared to direct transmission. Moreover, numerical examples show that the relay location has a significant impact on the EE performance.
\end{abstract}

\section{INTRODUCTION}

Recently, wireless body area networks (WBANs) have attracted much attention in telemedicine and mhealth [1]. Ultrawideband (UWB) is a potential technology for applications in WBANs, owing to its low-power operation. In UWB based implant BANs (IBANs), the power resources of implant nodes are very limited and batteries are not rechargeable and replaceable. Thus, one of the most critical challenges for UWB based IBANs is energy efficiency (EE). On the other hand, cooperative communication techniques have gained much attention in wireless sensor networks (WSNs) which can be used to improve the EE effectively [2]-[4].

Different from conventional WSNs, UWB based WBANs have some unique properties, e.g., analog transmission, distinct channel characteristics, and very small network size. Therefore, it is not straightforward to apply the existing strategies in [2]-[4] to UWB based WBANs. Thus, research into cooperative transmission has been conducted in WBANs regarding the EE [5]-[8]. In [5], the optimal joint power allocation and coordinator deployment were derived to minimize the power consumption for various system requirements in WBANs. In [6], the EE of cooperative transmission was studied from a relay selection perspective for UWB based WBANs. In [7], the EE in UWB based IBANs is evaluated and the corresponding optimal relay location is discussed. In [8], a prediction-based relay selection scheme was proposed to improve the EE and transmission reliability. However, all aforementioned work only focused on the single relay case in WBANs. In fact, the EE of cooperative transmission with multiple relays in UWB based IBANs is still an open issue.

On the other hand, the signal strength is mostly affected by the physical location of the nodes in relation to each other as well as the human body in WBANs. In particular, both "in-body to on-body" and "on-body to on-body" channels are involved in cooperative IBANs, which makes the relay location have a critical impact on the system performance. Thus, it is important to investigate the $\mathrm{EE}$ of cooperative transmission with multiple relays and evaluate the impact of relay location in UWB based IBANs.

In this paper, a distributed beamforming problem for the EE optimization is presented in UWB based IBANs. Specifically, an IBAN consisting of one implanted transmitter, several parallel wearable relays, and one body network coordinator is considered. To evaluate the impact of relay location on the EE, a relay location based cooperative network model is developed. With the proposed model, the EE optimization problem is mathematically formulated with an individual power constraint on each relay, which is equivalent to solving a distributed power allocation problem, where each relay properly adjusts its own power to make the system EE maximized. Since the problem is a non-convex and nonlinear problem, sequential quadratic programming (SQP) algorithm combined with scatter search are exploited to find the global optimum. Simulations results show that the proposed beamforming scheme is superior to other transmission schemes regarding the EE. A considerable improvement can be achieved not only in EE but also in spectral efficiency (SE) compared to direct transmission, which indicates that the proposed scheme is an effective way to prolong the lifetime of IBANs and extend the transmission range in IBANs. Moreover, the relay location plays an important role on the EE performance and our numerical examples reveal that the optimal relay location varies with the battery power limit of the implant node.

\section{System And Channel Models}

\section{A. System Scenarios}

As shown in Fig. 1, a cooperative model for IBANs is proposed, which consists of an implant node $S$ (Source), $m(m \geq 2)$ wearable relay nodes $\left(R_{i}\right.$, for $\left.\mathrm{i}=1, \ldots, m\right)$ and a 
body network coordinator $D$ (Destination). In this paper, a two-phase amplify-and-forward (AF) transmission protocol is studied. In the first phase, $S$ broadcasts its signal to relays, and during the second phase, relays forward their received signals to $D$. In Fig. $1, d_{S D}$ and $d_{r}$ denote the distances from $S$ to $D$ and penetration depth from $S$ to the body surface, respectively. On the body surface, a xy-plane is constructed to present coordinates of $m$ relays, where $P$ represents the projection of $S$ on the body surface and it is set to be the origin point, and the $\mathrm{x}$-axis is along $P$ to $D$. Throughout this paper, a special parallel relay topology is considered, where $m$ relays have the same $x$-coordinate $x_{0}[\mathrm{~mm}]\left(x_{0} \geq 0\right)$ and are evenly distributed along y-axis. With a given $y$-coordinate $y_{i}[\mathrm{~mm}]$ for relay $i$, we have

$$
d_{S R_{i}}=\sqrt{x_{0}^{2}+y_{i}^{2}+d_{r}^{2}},
$$

and

$$
d_{R_{i} D}=\sqrt{\left(x_{0}-d_{S D} \sin \theta\right)^{2}+y_{i}^{2}},
$$

where $d_{S R_{i}}$ and $d_{R_{i} D}$ are the distances from $S$ to relay $i$ and relay $i$ to $D$, respectively. $\theta=\arccos \left(d_{r} / d_{S D}\right)$.

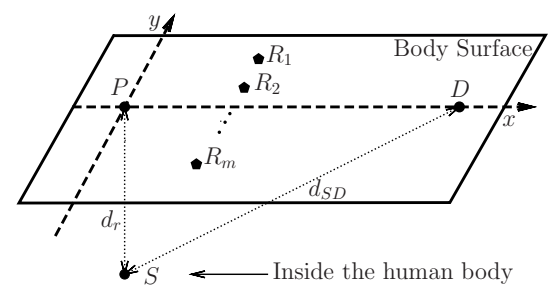

Fig. 1. Proposed cooperative model for IBANs.

\section{B. Channel Models}

In UWB based IBANs, two types of channel models are considered for the two-phase cooperative transmission, namely "along-torso" channel model from relays to $D$ and "in-body" channel model from $S$ to relays. Table I summarizes the path loss models with the corresponding parameters [9], [10].

TABLE I

UWB BASED BAN PATH LOSS MODELS

\begin{tabular}{|c|c|}
\hline "along-torso" channel & $P L_{0}^{d B}(d)=P_{0}+10 n_{0} \log _{10}\left(\frac{d}{d_{r 0}}\right)$ \\
\hline$P_{0}[\mathrm{~dB}]$ & 44.6 \\
\hline$n_{0}$ & 3.1 \\
\hline$d_{r 0}[m]$ & 0.1 \\
\hline \hline "in-body" channel & $P L_{1}^{d B}(d)=P_{1}+a\left(d / d_{r 1}\right)^{n_{1}}+H$ \\
\hline$P_{1}[\mathrm{~dB}]$ & 10 \\
\hline$a$ & 0.987 \\
\hline$n_{1}$ & 0.85 \\
\hline$d_{r 1}[m]$ & 0.001 \\
\hline
\end{tabular}

${ }^{1} H$ is a Gaussian distributed random variable with zero mean and standard deviation 7.84 .

Accordingly, the path losses in the linear scale from $S$ to $R_{i}$ and $R_{i}$ to $D$ can be obtained as,

$$
P L_{S R_{i}}\left(d_{S R_{i}}\right)=10^{P L_{1}^{d B}\left(d_{S R_{i}}\right) / 10}, \quad i=1, \ldots, m
$$

and

$$
P L_{R_{i} D}\left(d_{R_{i} D}\right)=10^{P L_{0}^{d B}\left(d_{R_{i} D}\right) / 10}, \quad i=1, \ldots, m
$$

respectively.

For all the links considered, the energy-normalized channel impulse response (CIR) can be written as

$$
h_{k}(t)=\sum_{l=0}^{L_{k}-1} \alpha_{l, k} \delta\left(t-\tau_{l, k}\right),
$$

where $k \in\left\{S R_{i}, R_{i} D\right\}$ denotes the links from $S$ to $R_{i}$ and $R_{i}$ to $D$ respectively. $L_{k}$ is the number of multipaths, $\tau_{l, k}$ is the delay of the $l$ th path, and $\alpha_{l, k}$ is the gain of the $l$ th path. Since real signals are employed in UWB systems, each path gain is real also. Further detail on the delay profile for "along-torso" and "in-body" links can be found in [9] and [10], respectively.

\section{Distributed Transmission Scheme}

Without loss of generality, we present the IR-UWB signal transmission with pulse-amplitude modulation (PAM). When data is modulated, pulse shaped and transmitted repeatedly over $N_{f}$ consecutive frames at $S$, the received signal at relay $i$ is given by

$$
\begin{aligned}
r_{S R_{i}}(t)= & \sqrt{\frac{P_{s}}{P L_{S R_{i}}\left(d_{S R_{i}}\right)}} \sum_{j=0}^{N_{f}-1} g_{S R_{i}}\left(t-j T_{f}\right) \\
& +n_{S R_{i}}(t), \quad b= \pm 1
\end{aligned}
$$

where $b$ is the transmitted symbol and $P_{s}$ is the transmit power of the implant node and the value of $P_{s}$ depends on the battery power limit of the implant node.

$$
g_{S R_{i}}(t)=\omega(t) * h_{S R_{i}}(t)=\sum_{l=0}^{L_{S R_{i}}-1} \alpha_{l, S R_{i}} \omega\left(t-\tau_{l, S R_{i}}\right),
$$

where $*$ represents convolution. $\omega(t)$ denotes the ultrashort pulse waveform with $T_{w}$ duration, which has the unit energy $\int_{t=0}^{T_{f}} \omega^{2}(t) d t=1 . T_{f}$ is the duration of frame and it is set to be large enough to avoid the inter-symbol interference (ISI). $n_{S R_{i}}(t)$ is the additive white Gaussian noise (AWGN) with zero mean and variance $\sigma^{2}$.

At each relay, a received pulse waveform matched filter is employed. After summing up all the outputs over $N_{f}$ frames, the decision statistic $b$ at relay $i$ can be written as

$$
b_{R_{i}}=b N_{f} \sqrt{\frac{P_{s}}{P L_{S R_{i}}\left(d_{S R_{i}}\right)}} \xi_{T_{f}}\left(h_{S R_{i}}\right)+\hat{n}_{S R_{i}},
$$

where $\xi_{T_{f}}\left(h_{S R_{i}}\right)$ is the captured multipath energy during $T_{f}$ at relay $i$ and $\hat{n}_{S R_{i}}$ is a white Gaussan noise with zero mean and variance $N_{f} \xi_{T_{f}}\left(h_{S R_{i}}\right) \sigma^{2}$.

With $\left\{b_{R_{i}}\right\}_{i=1}^{m}$, distributed space-time block coding (STBC) is applied at relays to achieve spatial diversity gain. Let $\mathbf{B}$ be the STBC matrix with dimension $m \times N_{f}^{\prime}$, where $N_{f}^{\prime}$ is the block length. Examples of the STBC matrix are shown in Table II. In this paper, we assume that $N_{f}=Q N_{f}^{\prime}$ and $Q$ is 
an integer. Thus, $N_{f}$ frames are divided into $Q$ frame blocks and the transmit signal vector at relays can be modeled as

$$
\mathbf{t}_{R}(t)=\sqrt{P_{\max }} \sum_{q=1}^{Q} \hat{\mathbf{b}}_{R} \hat{\mathbf{K}}_{R} \hat{\mathbf{W}}_{R} \mathbf{B} \boldsymbol{\omega}\left(t-N_{f} T_{f}, q\right),
$$

where $\mathbf{t}_{R}(t)=\left[t_{R_{1}}(t), t_{R_{2}}(t), \ldots, t_{R_{m}}(t)\right]^{T}$ and the element $t_{R_{i}}(t)$ represents the transmitted signal at relay $i . P_{\max }$ is the maximum transmit power at each relay, which is constrained by Federal Communications Commission (FCC) power spectral density (PSD) emission limit for UWB signals. $\hat{\mathbf{b}}_{R} \triangleq$ $\operatorname{diag}\left\{b_{R_{1}}, \ldots, b_{R_{m_{2}}}\right\} . \hat{\mathbf{K}}_{R} \triangleq \operatorname{diag}\left\{\left|b_{R_{1}}\right|^{-1}, \ldots,\left|b_{R_{m}}\right|^{-1}\right\}$ and $\left|b_{R_{i}}\right|=\left(\frac{N_{f}^{2} P_{s} \xi_{T_{f}}^{2}\left(h_{S R_{i}}\right)}{P L_{S R_{i}}\left(d_{S R_{i}}\right)}+N_{f} \xi_{T_{f}}\left(h_{S R_{i}}\right) \sigma^{2}\right)^{\frac{1}{2}} \cdot \hat{\mathbf{W}}_{R} \triangleq$ $\operatorname{diag}\left\{w_{1}, \ldots, w_{m}\right\}$ is the diagonal matrix of the relay power allocation weights and $0 \leq w_{i} \leq 1$. Clearly, the transmit power of relay $i$ is $w_{i}^{2} P_{\max }$. We define $\boldsymbol{\omega}(t, q) \triangleq[\omega(t-$ $\left.N_{f}^{\prime}(q-1) T_{f}\right), \omega\left(t-N_{f}^{\prime}(q-1) T_{f}-T_{f}\right), \ldots, \omega\left(t-N_{f}^{\prime}(q-\right.$ 1) $\left.\left.T_{f}-\left(N_{f}^{\prime}-1\right) T_{f}\right)\right]^{T}$ is the waveform vector with length $N_{f}^{\prime}$.

TABLE II

EXAMPLES OF STBC MATRIX B FOR RELAYS

\begin{tabular}{|c|c|c|}
\hline$m$ & $N_{f}^{\prime}$ & B \\
\hline \hline 2 & 2 & $\left(\begin{array}{cc}1 & -1 \\
1 & 1\end{array}\right)$ \\
\hline 3 & 4 & $\left(\begin{array}{cccc}1 & -1 & -1 & -1 \\
1 & 1 & 1 & -1 \\
1 & -1 & 1 & 1\end{array}\right)$ \\
\hline
\end{tabular}

At $D$, a Rake receiver with $L_{r}$ fingers is employed and the delayed reference waveforms $\left\{\omega\left(t-\tau\left(l_{r}\right)\right)\right\}_{l_{r}=0}^{L_{r}-1}$ are used. After combining the Rake outputs over $N_{f}^{\prime}$ frames in each frame block, the output per frame block can be written as

$$
\begin{aligned}
z_{D} & =\sqrt{P_{\max }} \sum_{l_{r}=0}^{L_{r}-1} \boldsymbol{\beta}_{R D}^{T}\left(l_{r}\right) \mathbf{B B}^{T} \hat{\mathbf{K}}_{R} \hat{\mathbf{W}}_{R} \hat{\mathbf{P}} \hat{\mathbf{L}}_{R D} \mathbf{b}_{R}+\bar{n}_{D}(t) \\
& =N_{f}^{\prime} \sqrt{P_{\max }} \boldsymbol{\xi}_{R D}^{T} \hat{\mathbf{K}}_{R} \hat{\mathbf{W}}_{R} \hat{\mathbf{P L}}{ }_{R D} \mathbf{b}_{R}+\bar{n}_{D}
\end{aligned}
$$

where $\mathbf{b}_{R}=\left[b_{R_{1}}, \ldots, b_{R_{m}}\right]^{T} . \quad \boldsymbol{\beta}_{R D}\left(l_{r}\right) \triangleq$ $\left[\beta_{R_{1} D}^{2}\left(l_{r}\right), \ldots, \beta_{R_{m} D}^{2}\left(l_{r}\right)\right]^{T} \cdot \beta_{R_{i} D}\left(l_{r}\right)=\int_{0}^{T_{f}} g_{R_{i} D} \omega(t-$ $\left.\tau\left(l_{r}\right)\right) d t \quad$ and $\quad g_{R_{i} D}(t)=\omega(t) * h_{R_{i} D}(t)$. $\hat{\mathbf{P L}}_{R D} \triangleq \operatorname{diag}\left\{P L_{R_{1} D}^{-\frac{1}{2}}\left(d_{R_{1} D}\right), \ldots, P L_{R_{m} D}^{-\frac{1}{2}}\left(d_{R_{m} D}\right)\right\}$. $\boldsymbol{\xi}_{R D} \triangleq\left[\xi_{R_{1} D}, \ldots, \xi_{R_{m} D}\right]^{T}$ and $\xi_{R_{i} D}=\sum_{l_{r}=0}^{L_{r}-1} \beta_{R_{i} D}^{2}\left(l_{r}\right)$. Notice that $\xi_{R_{i} D}$ represents the captured multipath energy from link $R_{i}$ to $D . \bar{n}_{D}$ is the AWGN with zero mean and variance $N_{f}^{\prime} \sum_{i=1}^{m} \xi_{R_{i} D} \sigma^{2}$.

Combining all outputs over $Q$ frame blocks and substituting
(3) into (5), the decision variable for $b$ at $D$ can be given by

$$
\begin{aligned}
\hat{z} & =N_{f} \sqrt{P_{m a x}} \boldsymbol{\xi}_{R D}^{T} \hat{\mathbf{K}}_{R} \hat{\mathbf{W}}_{R} \hat{\mathbf{P L}} \hat{\mathbf{L}}_{R D} \mathbf{b}_{R}+\hat{n}_{D} \\
& =\underbrace{b N_{f}^{2} \sqrt{P_{m a x} P_{s}} \boldsymbol{\xi}_{R D}^{T} \hat{\mathbf{K}}_{R} \hat{\mathbf{W}}_{R} \hat{\mathbf{P L}}_{R D} \hat{\mathbf{P L}}_{S R} \boldsymbol{\xi}_{S R}}_{\hat{z}_{s}} \\
& +\underbrace{N_{f} \sqrt{P_{\max }} \boldsymbol{\xi}_{R D}^{T} \hat{\mathbf{K}}_{R} \hat{\mathbf{W}}_{R} \hat{\mathbf{P L}} \hat{\mathbf{L}}_{R D} \hat{\mathbf{n}}_{S R}}_{\hat{z}_{n_{S R}}}+\underbrace{\hat{n}_{D}}_{\hat{z}_{n_{R D}}},
\end{aligned}
$$

where $\hat{\mathbf{P L}}_{S R} \triangleq \operatorname{diag}\left\{P L_{S R_{1}}^{-\frac{1}{2}}\left(d_{S R_{1}}\right), \ldots, P L_{S R_{m}}^{-\frac{1}{2}}\left(d_{S R_{m}}\right)\right\}$ and $\boldsymbol{\xi}_{S R} \triangleq\left[\xi_{T_{f}}\left(h_{S R_{1}}\right), \ldots, \xi_{T_{f}}\left(h_{S R_{m}}\right)\right]^{T} . \quad \hat{\mathbf{n}}_{S R} \triangleq$ $\left[\hat{n}_{S R_{1}}, \ldots, \hat{n}_{S R_{m}}\right]^{T} . \hat{n}_{D}$ is the AWGN with zero mean and variance $N_{f} \sum_{i=1}^{m} \xi_{R_{i} D} \sigma^{2}$. In (6), $\hat{z}_{s}, \hat{z}_{n_{S R}}$, and $\hat{z}_{n_{R D}}$ are defined as the useful signal, noise from the first hop, and noise from the second hop, respectively.

With (6), the EE [bits/Joule/Hz] for the distributed beamforming transmission can be defined as

$$
U_{S R D} \triangleq \frac{r_{S R D}}{P_{S R D}}
$$

where

$$
r_{S R D}=\frac{1}{2 N_{f}} \log _{2}\left(1+\frac{\mathrm{E}\left\{\left|\hat{z}_{s}\right|^{2}\right\}}{\mathrm{E}\left\{\left|\hat{z}_{n_{S R}}\right|^{2}\right\}+\mathrm{E}\left\{\left|\hat{z}_{n_{R D}}\right|^{2}\right\}}\right)
$$

is the $\mathrm{SE}[\mathrm{bits} / \mathrm{s} / \mathrm{Hz}]$ for cooperative transmission.

$$
P_{S R D}=\frac{1}{2}\left(P_{s}+\mathbf{w}_{R}^{T} \mathbf{w}_{R} P_{\text {max }}+(m+1)\left(P_{c t}+P_{c r}\right)\right)
$$

is the power dissipation for cooperative transmission per frame. $\mathbf{w}_{R} \triangleq\left[w_{1}, \ldots, w_{m}\right]^{T}$. $P_{c t}$ and $P_{c r}$ are the power dissipations for transmission circuit and receiver circuit per frame, respectively. In this paper, it is assumed that $P_{c t}$ and $P_{c r}$ are constant for each node.

\section{Optimal Energy EfFiciency}

In this section, we formulate the distributed beamforming problem for the EE optimization. Since the problem is nonconvex, SQP algorithm combined with scatter search are exploited to find the global optimum.

Using (6), we have

$$
\mathrm{E}\left\{\left|\hat{z}_{s}\right|^{2}\right\}=\mathbf{w}_{R}^{T} \mathbf{O}_{s} \mathbf{w}_{R}
$$

where $\mathbf{O}_{s}=N_{f}^{4} P_{\max } P_{s} \hat{\boldsymbol{\xi}}_{R D}^{2} \hat{\mathbf{K}}_{R}^{2} \hat{\mathbf{P L}}_{R D}^{2} \hat{\mathbf{P L}}_{S R}^{2} \boldsymbol{\xi}_{S R} \boldsymbol{\xi}_{S R}^{T}$. $\hat{\boldsymbol{\xi}}_{R D} \triangleq \operatorname{diag}\left\{\xi_{R_{1} D}, \ldots, \xi_{R_{m} D}\right\}$.

$$
\mathrm{E}\left\{\left|\hat{z}_{n_{S R}}\right|^{2}\right\}=\mathbf{w}_{R}^{T} \mathbf{O}_{n} \mathbf{w}_{R}
$$

where $\mathbf{O}_{n}=N_{f}^{3} P_{\max } \sigma^{2} \hat{\boldsymbol{\xi}}_{R D}^{2} \hat{\mathbf{K}}_{R}^{2} \hat{\mathbf{P L}}_{R D}^{2} \hat{\boldsymbol{\xi}}_{S R}$ and $\hat{\boldsymbol{\xi}}_{S R} \triangleq$ $\operatorname{diag}\left\{\xi_{T_{f}}\left(h_{S R_{1}}\right), \ldots, \xi_{T_{f}}\left(h_{S R_{m}}\right)\right\}$. And

$$
\mathrm{E}\left\{\left|\hat{z}_{n_{R D}}\right|^{2}\right\}=N_{f} \sum_{i=1}^{m} \xi_{R_{i} D} \sigma^{2} .
$$

Substituting (10)-(12) into (8) and using (9), the EE optimization problem can be written as

$$
\begin{array}{ll}
\underset{\mathbf{w}_{R}}{\operatorname{maximize}} & \frac{r_{S R D}\left(\mathbf{w}_{R}\right)}{P_{S R D}\left(\mathbf{w}_{R}\right)} \\
\text { subject to } & \mathbf{0}_{m} \preceq \mathbf{w}_{R} \preceq \mathbf{I}_{m},
\end{array}
$$


where

$r_{S R D}\left(\mathbf{w}_{R}\right)=\frac{1}{2 N_{f}} \log _{2}\left(1+\frac{\mathbf{w}_{R}^{T} \mathbf{O}_{s} \mathbf{w}_{R}}{\mathbf{w}_{R}^{T} \mathbf{O}_{n} \mathbf{w}_{R}+N_{f} \sum_{i=1}^{m} \xi_{R_{i} D} \sigma^{2}}\right)$.

$\mathbf{0}_{m}$ is a $m$-dimensional vector with all zero entries and $\mathbf{I}_{m}$ is a $m$-dimensional vector with all one entries. With two $m$ dimensional vectors $\tilde{\mathbf{a}}$ and $\tilde{\mathbf{c}}, \tilde{\mathbf{a}} \preceq \tilde{\mathbf{c}}$ means $\tilde{a}_{i} \leq \tilde{c}_{i}$ for all $i \in[1, m]$.

Using (14) and (9), we can see that the problem in (13) is a non-convex and nonlinear programming problem and the corresponding Karush-Kuhn-Tucker (KKT) equations are only necessary for the optimal solution. In this paper, SQP algorithm and scatter search are employed to find the global optimal solution $\mathbf{w}_{R}^{o}$.

SQP algorithm [11] is a powerful iterative method for nonconvex and nonlinear optimization. At each major iteration, an approximation is made of the Hessian of the Lagrangian function using a quasi-Newton updating method. This is then used to generate a QP subproblem whose solution is used to form a search direction for a line search procedure. Within finite iterations, SQP algorithm will converge to a local optimum. On the other hand, scatter search is a global optimization algorithm, which can find the optimal solution effectively by calling the SQP algorithm several times with different starting points. Unfortunately, we are unable to provide the close-form expression of $\mathbf{w}_{R}^{o}$ since the optimal solution can only be found numerically. In our simulations, Matlab's optimization toolbox is used to implement the SQP algorithm.

\section{Simulation}

To evaluate the EE of the proposed distributed beamforming scheme in UWB based IBANs, numerical results and examples are presented in this section. System parameters are given in Table III. In addition, the noise PSD is set to be $-174 \mathrm{dBm} / \mathrm{Hz}$

TABLE III

SYSTEM PARAMETERS

\begin{tabular}{|c|c|c|}
\hline$m=2$ & $N_{f}=4$ & $T_{w}=0.7 \mathrm{~ns}$ \\
\hline$T_{f}=100 \mathrm{~ns}$ & $d_{S D}=200 \mathrm{~mm}$ & $d_{r}=50 \mathrm{~mm}$ \\
\hline$L_{r}=4$ & $P_{c t}=100 \mu \mathrm{W}$ & $P_{c r}=200 \mu \mathrm{W}$ \\
\hline
\end{tabular}

and the system bandwidth is $1.4 \mathrm{GHz}$. Since the average FCC PSD emission limit for UWB signals is $-41.3 \mathrm{dBm} / \mathrm{MHz}$, the maximum average transmit power $P_{\text {ave }}$ is $-9.8 \mathrm{dBm}$. With the duty cycle $T_{w} / T_{f}, P_{\max }=P_{\text {ave }} * T_{f} / T_{w} \approx 12 \mathrm{dBm}$. For the transmit power of the implant node, we set $P_{s} \leq 10 \mathrm{dBm}$ considering the emission limit and safety inside the human body. At relays, we assume that $\xi_{T_{f}}\left(h_{S R_{i}}\right) \approx 1$ for all $i \in$ $[1, m]$ without considering the ISI. At $D$, a selective Rake receiver is employed to combine the $L_{r}$ strongest multipath components. Every simulation result in this section is averaged over 1000 realizations.

Fig. 2 shows the average EE versus $P_{s}$ in a 2-relay UWB based IBAN. For this case, the coordinates of the two relays are set to be $\{(90,20)\}$ and $\{(90,-20)\}$, respectively. Four

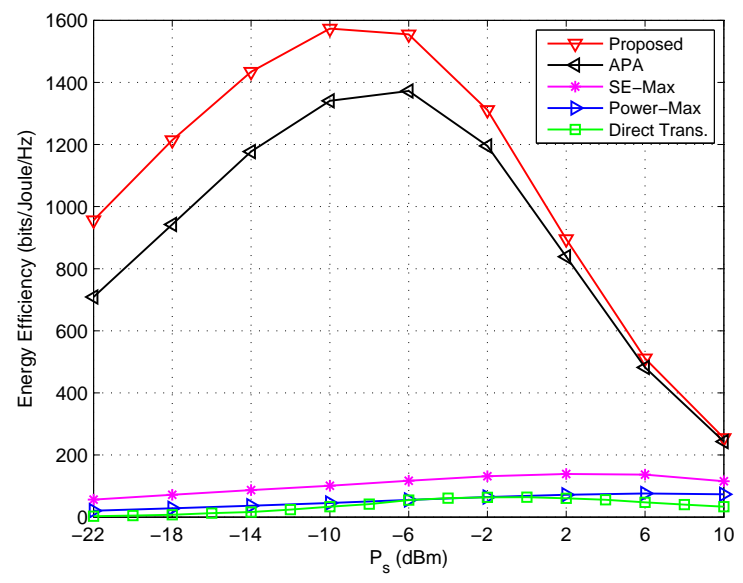

Fig. 2. Average EE versus $P_{s}$ in a 2-relay network.

transmission schemes are illustrated in this figure to compare with the proposed beamforming scheme, which are the average power allocation (APA) scheme, the power-maximizing (Power-Max) scheme (every relay uses its maximum power), the SE-maximizing (SE-Max) scheme and direct transmission, respectively. In the APA scheme, the power allocation weight for the relay $i$ is set to be $\mathrm{E}\left\{w_{i}^{o}\right\}$, which denotes the average transmit power consumed by the relay $i$ in the proposed beamforming scheme. Thus, the APA scheme uses the same amount of power resource as the proposed scheme. In the SEMax scheme, $\mathbf{w}_{R}$ is obtained to maximize the SE in (14), which needs the same channel information as the proposed scheme. As shown in this figure, the proposed distributed beamforming scheme is superior to all the other schemes in terms of the EE. For the APA scheme, its performance is only slightly inferior to that of the proposed scheme, especially when $P_{s}$ is in the moderate-to-high regimes. For the PowerMax and SE-Max schemes, both of them cannot achieve a satisfactory performance, which results from the fact that employing large transmit power in both schemes is deleterious to the EE. Compared to direct transmission, we can see that the proposed beamforming scheme can provide a substantial performance improvement. This evidence indicates that the lifetime of IBANs can be prolonged considerably by using the proposed distributed beamforming scheme.

Fig. 3 depicts the average $\mathrm{SE}$ versus $P_{s}$ in a 2-relay network. The coordinates of the two relays are the same as those in Fig. 2. Obviously, the SE-Max scheme has the best performance in terms of the SE. Although the performance gap between the proposed scheme and the SE-Max scheme grows larger as $P_{s}$ increases, our proposed scheme still have an acceptable SE, which outperforms the APA scheme with the same power resource and direct transmission.

In Fig. 4, the average EE versus $d_{S D}$ is illustrated. To make a fair comparison, the coordinates of the two relays are fixed at $\{(0,20)\}$ and $\{(0,-20)\}$, respectively. We notice that direct transmission is not feasible to attain an energy efficient transmission in UWB based IBANs for large $d_{S D}$. That is 


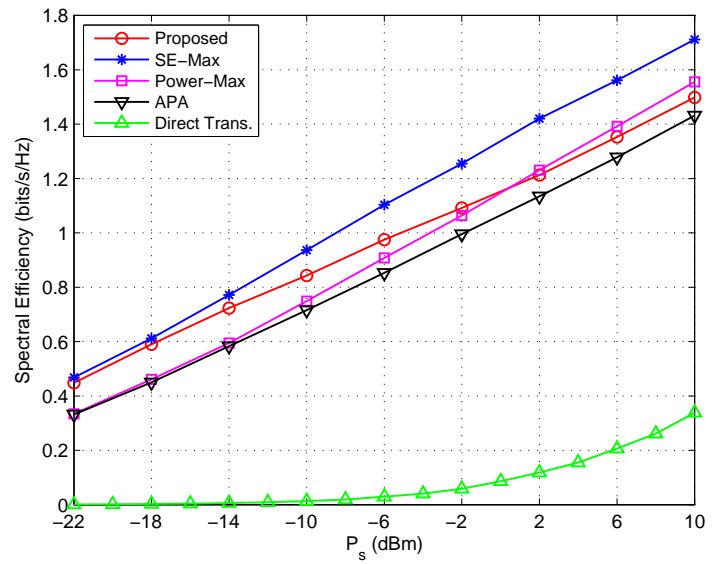

Fig. 3. Average SE versus $P_{s}$ in a 2-relay network.

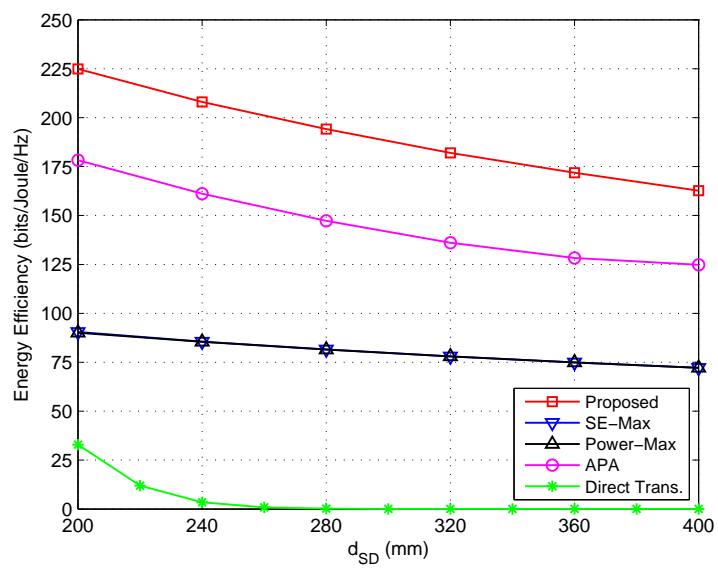

Fig. 4. Average EE versus $d_{S D}$ in a 2-relay network.

to say, without the line of sight between $S$ and $D$, the EE of direct transmission is affected adversely due to the severe propagation conditions within the human body. By contrast, the proposed scheme exhibits a weak dependence upon $d_{S D}$, which reveals that the proposed scheme can be an effective way for the extension of transmission range in IBANs.

To investigate the impact of relay location, Fig. 5 presents the average EE versus $P_{s}$ with different relay locations in a 2-relay network, where the y-coordinate of the two relays are fixed at $\{20\}$ and $\{-20\}$, respectively. For the $\mathrm{x}$-coordinate, we assume that $x_{0}$ has the value of 0,90 , and 150 , respectively, where $x_{0}=0$ represents that relays are close to $P, x_{0}=90$ represents that relays are located near the middle of $P$ and $D$, and $x_{0}=150$ represents that relays are close to $D$. Based on the observations, the EE performance of the proposed scheme is sensitive to the relay location with a given $P_{s}$. When the battery power of $S$ is very limited, employing the relays close to $P$ can achieve the utmost EE. As $P_{s}$ becomes larger, choosing the relays close to the middle point of $P$ and $D$ is the best option.

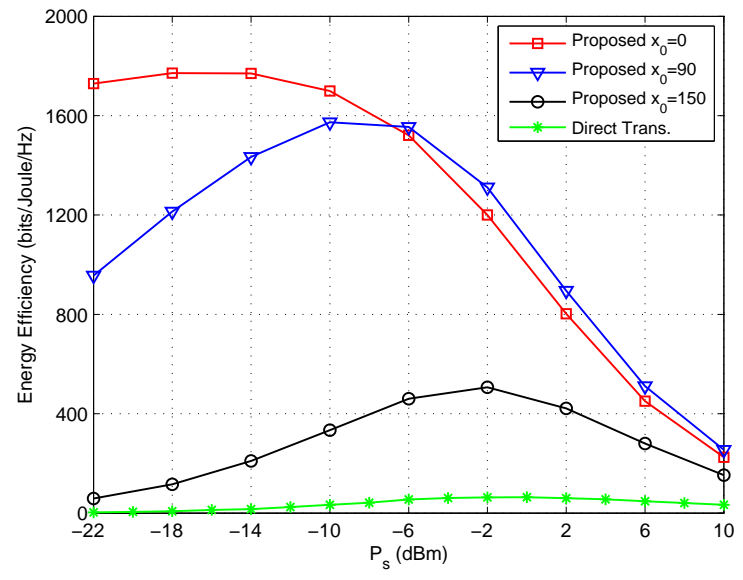

Fig. 5. Average EE versus $P_{s}$ with different relay locations.

\section{CONCLUSIONS}

In this paper, the EE optimization problem in relay UWB based IBANs is studied. We proposed a distributed beamforming scheme to maximize the system EE. Numerical examples demonstrate that the proposed scheme is an effective way to prolong the lifetime of IBANs and extend the transmission range in IBANs. We further show that the relay location is an influential parameter in UWB based IBANs. The EE with different relay locations may have a notable difference. Thus, the effect of relay location cannot be ignored.

\section{REFERENCES}

[1] M. Patel and J. Wang, "Applications, challenges, and prospective in emerging body area networking technologies," IEEE Wireless Coтmunications, vol. 17, no. 1, 2010, pp. 80-88.

[2] S. Cui, A. J. Goldsmith, and A. Bahai, "Energy-efficiency of MIMO and cooperative MIMO in sensor networks," IEEE Journal on Selected Areas in Communications, vol. 22, no. 6, Aug. 2004, pp. 1089-1098.

[3] Y. Hao, Y. Jing, and S. ShahbazPanahi, "Energy efficient network beamforming design using power-normalized SNR," IEEE Transactions on Wireless Communications, vol. 13, no. 5, May 2014, pp. 2756-2769.

[4] H. Kim, S-R. Lee, C. Song, and I. Lee, "Optimal power allocation for energy efficiency maximization in distributed antenna system," IEEE ICC, June 2013, pp. 4362-4366.

[5] C. Su, P. Wang, and R. Chai, "Joint power allocation and coordinator deployment for wireless body area network," IEEE WCSP, Oct. 2012, pp. $1-6$

[6] J. Ding, E. Dutkiewicz, X. J. Huang, and G. F. Fang, "Energy-efficient cooperative relay selection for UWB based body area networks," IEEE ICUWB, Sep. 2013, pp. 97-102.

[7] J. Ding, E. Dutkiewicz, and X. Huang, "Energy efficient cooperative communication for UWB based in-body area networks," ACM Bodynets, Sep. 2013, pp. 29-34.

[8] F. Hui, L. Bin, Z. Yan, C. Zhang, and C. Chen, "Prediction-based dynamic relay transmission scheme for wireless body area networks," IEEE PIMRC, Sep. 2013, pp. 2539-2544.

[9] K. Y. Yazdandoost and K. Sayrafian-Pour, "Channel model for body area network (ban)," IEEE P802.15 Working Group for Wireless Personal Area Networks (WPANs), Tech. Rep. Document IEEE802.15-08-078005-0006, February 2009.

[10] A. Khaleghi, R. Chvez-Santiago, and I. Balasingham, "Ultra-wideband statistical propagation channel model for implant sensors in the human chest," IET Microwaves, Antennas and Propagation, vol. 5, no. 15, Dec. 2011, pp. 1805-1812.

[11] J. Nocedal and S. J. Wright, Numerical Optimization. Springer, 1999. 